\title{
Magic Mathematics Based on New Matrix Transformations (2D and 3D) for Interdisciplinary Physics, Mathematics, Engineering and Energy Management
}

\author{
Prof. Dr.-Ing. Wolfram Stanek ${ }^{1,2}$ and Dipl. Ing. Maralo Sinaga ${ }^{3}$ \\ 1 University of Applied Sciences Koblenz, \\ ${ }^{2}$ Guest Lecturer at Swiss German University, BSD City-Jakarta \\ ${ }^{3}$ Head of Mechatronics Department, Swiss German University, BSD City-Jakarta \\ ${ }^{1}$ Germany \\ 2,3Indonesia
}

\section{Introduction}

Mathematics is magic. If we can either use one formula for a wide range of applications or the formula itself will produce magic properties. As one of several introductory examples the generally not well known Leibniz formula for calculating determinants in matrix theory will show that both the well known Laplace laws and Sarrus rules for evaluating matrices are only graphically visualised subsets of this ingenious Leibniz formula. Visualising complex formulas and matrix transformations in $2 \mathrm{D}$ and $3 \mathrm{D}$ as equivalent graphs is a basic method of the main author in this publication. The huge range of fascinating technical applications based on 2D magic matrices will be sketched: Constant distribution in all directions of numbers, power, energies, element properties, transport, automation, information flows etc or compensation of punctual disturbances without variation of sum of energy or automatic minimization of energy loss remaining constant distribution or both concentration of energies in near field and hiding of energies in far field or solving magic equation systems in mathematics without using back tracking methods etc.

\section{Background}

The extremely complex problem in mathematics of finding a perfect solution of a $4 \times 4 \times 4$ - 3D magic cube (64 unknowns, but 76 equations/conditions) with constant sum in all directions and continuous numbers from 1 to 64 was solved first by the German mathematician W. Trump in the year 2004 (Spectrum of Science, 2008-2): But this world wide first solution of a $4 \times 4 \times 4$ magic cube was only based on parallel computations with several computers and extremely time-intensive back-tracking methods with time consuming solution. In contrast to this computer-based solution of $4 \times 4 \times 4$ magic cubes in 2004, the main author Prof. Dr. W. Stanek has shown a new analytical method manually solving this problem during a presentation on German MemoMasters 2008 and 2009: Using this analytical method for 4x4x4 
magic cubes, the manual 3D solution lasts a few minutes - applying this algorithm the solution time with MATLAB® needs only fractions of seconds (ca. $0.01 \mathrm{~s}$ ).

The results of these matrix transformations for magic 64-cells-cubes show two main aspects:

a. Extremely fast solution of such matrix problems in 3D by immediate transformation from magic 2D matrices to magic 3D cubes with remaining central magic properties.

b. New idea solving large sets of linear equations (with also determinant-zero-matrixproperty) NOT using conventional equation solvers (Gauss-Seidel, Newton-Raphson etc) and backtracking methods but only simplified geometrical 3D transformations and logic. This magic math algorithm is shown by visualised graph transformations and underlying equivalent structures.

\section{Magic square and magic cube (2800 B.C - 2008)}

Magic squares were known to Chinese mathematicians, and Arab mathematicians, possibly as early as the 7th century, when the Arabs conquered northwestern parts of the Indian subcontinent and to learned Indian mathematicians and astronomers, including other aspects of combinatorial mathematics. The most famous 2D magic squares are the Lo Shu square and the Duerer square.

A normal magic square contains the integers from 1 to $n^{2}$. The constant sum in every row, column and diagonal is called the magic constant or magic sum, $S$. The magic constant of a normal magic square with continuous numbers depends only on $\boldsymbol{n}$ and has the value:

$$
S=\frac{n}{2}\left(n^{2}+1\right)
$$

\subsection{Lo Shu Square}

Lo Shu square or the Nine Halls Diagram is the unique normal magic square of order $3 \times 3$. Lo Shu is part of the legacy of the most ancient Chinese mathematical and divinator traditions, and is an important emblem in "Feng Shui" the art of geomancy concerned with the placement of objects in relation to the flow of 'natural energy'.

The Lho Shu square was introduced in 2800 BC. Fig. 1.(a) shows the Loh Shu square used symbolism instead of numbers, and Fig. 1.(b) representing continuous number 1 to 9 this square. The Loh Shu square dimension is $n=3$, then the magic sum $S$ is 15 .

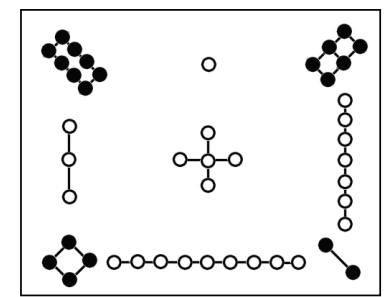

(a) Loh Shu square with symbols

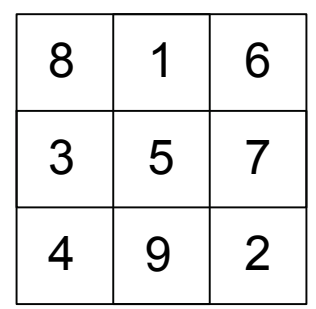

(b) Loh Shu square with numbers

Fig. 1. Loh Shu square (Wikipedia, 2010) 


\subsection{Duerer magic matrix}

The Renaissance engraving "Melancholia I" was developed by the German artist, painter, and mathematician Albrecht Duerer (in the year 1514). This image is filled with mathematical symbolism and in the upper right corner of the first picture a square can be seen. The Fig. 2(a) shows an enlarged view of the Duerer's square cropped from the image. This square is known as a magic square and was believed by many in Duerer's time to have genuinely magical properties. It does turn out to have some fascinating characteristics worth exploring.

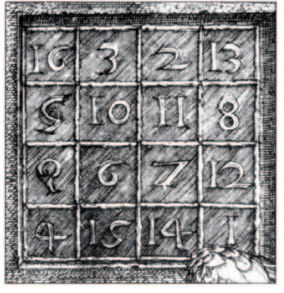

(a)

\begin{tabular}{|c|c|c|c|}
\hline 16 & 3 & 2 & 13 \\
\hline 5 & 10 & 11 & 8 \\
\hline 9 & 6 & 7 & 12 \\
\hline 4 & 15 & 14 & 1 \\
\hline
\end{tabular}

(b)

Fig. 2. Duerer square, Melancholia I, 1514, (Wikipedia, 2010)

The Duerer's square in Fig. 2(b) is filled up with continuous numbers 1 to 16. The square dimension is $n=4$, then the magic sum $S$ of Duerer's square is 34 .

\subsection{Sudoku}

Sudoku is a logic-based, combinatorial number-placement puzzle. The objective is to fill a $9 \times 9$ grid with digits so that each column, each row, and each of the nine $3 \times 3$ sub-grids that compose the grid contain all of the digits from 1 to 9 . The puzzle setter provides a partially completed grid, which typically has a unique solution.

Completed puzzles are always a type of Latin square with an additional constraint on the contents of individual regions. For example, the same single integer may not appear twice

- $\quad$ in the same 9x9 playing board row

- in the same 9x9 playing board column or

- $\quad$ in any of the nine $3 \times 3$ subregions of the 9x9 playing board.

The puzzle was popularized in 1986 by a Japanese puzzle company and became an international hit.

An extended example of the Sudoku game is shown in the Fig. 3, where a $9 \times 9$ square $\mathbf{A}$ is developed from a $3 \times 3$ square. Then the other cells are filled by using a regular shifting

\begin{tabular}{|c|c|c|c|c|c|c|c|c|c|c|c|c|c|c|c|c|c|c|c|c|c|c|c|c|c|c|c|c|}
\hline 2 & & 8 & 1 & 4 & & & 16 & & & 2 & & & & 11 & & 7 & 5 & & & 11 & 45 & 67 & 6 & $\sqrt{28}$ & 62 & 25 & 50 & 75 \\
\hline 1 & $\sqrt{4}$ & 7 & 3 & 61 & & 2 & 5 & 8 & & 6 & & & 2 & 9 & 4 & 6 & 11 & 18 & & 7 & 32 & 57 & 20 & $\sqrt{54}$ & $\sqrt{76}$ & 15 & 37 & 71 \\
\hline 3 & 6 & 9 & 2 & 5 & 8 & 1 & 4 & 18 & & 6 & 1 & 8 & 7 & 5 & 3 & 2 & 9 & $\sqrt{4}$ & & 24 & 46 & 80 & $\sqrt{16}$ & $\sqrt{41}$ & 66 & 2 & 36 & 58 \\
\hline 8 & 2 & 5 & 7 & 1 & 4 & 9 & 3 & 6 & & 9 & 4 & 2 & 1 & 8 & 6 & s & 3 & 7 & & 72 & 13 & 38 & 55 & 8 & 33 & 77 & 21 & 52 \\
\hline 7 & 1 & 4 & 9 & 3 & 6 & 8 & 2 & 5 & & 5 & 3 & 7 & 9 & 4 & 2 & 1 & 8 & 6 & & 59 & 3 & $\sqrt{34}$ & 81 & 22 & 47 & $\sqrt{64}$ & $\sqrt{17}$ & 42 \\
\hline 9 & $\sqrt{3}$ & 6 & 8 & 2 & 5 & 7 & 1 & 4 & & I & 8 & 0 & 5 & $\sqrt{3}$ & 7 & 9 & $\overline{4}$ & 2 & & $\sqrt{73}$ & 26 & S1 & 68 & 12 & 4 & 63 & 4 & 29 \\
\hline 5 & 8 & 2 & 4 & 7 & 1 & 6 & 9 & 13 & & 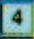 & 2 & & 8 & 6 & 1 & 3 & 7 & 5 & & 40 & 65 & 18 & 35 & $\sqrt{60}$ & 1 & 48 & 79 & 23 \\
\hline 4 & 7 & I & 6 & 9 & 3 & 5 & 8 & 2 & & 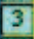 & 7 & 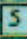 & 4 & 2 & 9 & 8 & 6 & 1 & & 30 & 61 & 5 & 49 & $\sqrt{74}$ & 27 & 44 & 69 & 10 \\
\hline$\sqrt{6}$ & 9 & 3 & 5 & 8 & 2 & 4 & 7 & ${ }^{\circ}$ & & 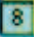 & 6 & & 3 & 7 & 5 & 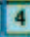 & 2 & 9 & & 53 & 78 & 19 & 39 & 70 & 14 & 31 & 56 & 9 \\
\hline
\end{tabular}

Fig. 3. Sudoku 9x9 from 3×3 square and bi-magic square (www.multimagie.com, 2009) 
method. From the square A, then square $\mathbf{B}$ is constructed by using rotating technique, and finally the bi-magic square $\mathbf{C}$ (with continuous number 1 to 81 ) is developed through addition of $\mathbf{A}$ and $\mathbf{B}$, example applying the formula $\mathbf{C}=\mathbf{9} \cdot(\mathbf{A}-\mathbf{1})+\mathbf{B}$. This magic square is bimagic (or multi-magic) if it remains magic after each of its numbers have been squared. This was introduced by Tarry and Cazalas. All cells content are squared, resulting in magic sums of $\mathrm{C}=369$ and $\mathrm{D}=20049$.

\section{Components of creative intelligence, Leibniz matrix and new solution technique.}

The following MATLAB® program cutoff to calculate the determinant of $n \times n$ matrix should be a central background of this publication. According to the famous Leibniz formula for determinant calculation of any $\mathrm{n} \times \mathrm{n}$ matrix, the fact shows that the Method of Sarrus and Method of Laplace to solve the determinant of the matrix use similar concept of the Leibniz formula with different visualisations. Both Sarrus and Laplace Methods could be structurised and visualised in mnemotechnique method by the main author. Finding the determinant of nxn matrix using the Leibniz formula is shown in the equation (2):

$$
\operatorname{det}(A)=\sum\left(\operatorname{sgn}(\sigma) \prod_{i=1}^{n} a_{i, \sigma(i)}\right)
$$

Based on this point, W.Stanek provides an algorithm to obtain any 3D magic structure, especially 64 cells cube, primarily the visualised structure of the algorithm. At the same time it will be shown that in a linear equation with a lot of unknowns (64 unknowns and 76 equations) is first solved by using the logical method and visual solution. This problem was first solved in 2004 by using computers, working in parallel and based on the back tracking algorithm method.

The equation is represented in MATLAB® function and algorithm shown as:

$\%$ MATLAB and Leibniz formula provide the same result

$\% \operatorname{det}(\mathrm{M} 1)=-360$ for $3 \times 3$ Loh Shu Matrix

$\%$ For magic $4 \times 4$ Duerer Matrix both methods yield $\operatorname{det}(\mathrm{M} 2)=0$, too

$\operatorname{det}$ A_Matlab $=\operatorname{det}($ M1)

$\%$ Leibniz-Formula for all nxn-Matrices, here only shown for $4 \times 4$-Matrix :

$\%$ With $\mathrm{n}=4$ follow $4 !=1 \times 2 \times 3 \times 4=24$ Terms for solutions of $\operatorname{det}(\mathrm{A})$

$\%$ With a14=0; a24=0; a34=0; a 44=1; a41=0; a42=0; a43=0;

$\%$ Leibniz formula also for 3x3-Matrices like i.e. magic 3×3 Loh Shu Matrix.

$\operatorname{det} A \_L e i b n i z=\left(a 11 * a 22 * a 33 * a 44+a 11 * a 23 * a 34 * a 42+a 1 *^{*} a 24 * a 32 * a 43 .\right.$.

- a11*a22*a34*a43 - a11*a23*a32*a44 - a11*a24*a33*a42...

+ a12*a21*a34*a43 + a12*a23*a31*a44 + a12*a24*a33*a41...

- a12*a21*a33*a44 - a12*a23*a34*a41 - a12*a24*a31*a43...

+ a13*a21*a32*a44 + a13*a22*a34*a41 + a13*a24*a31*a42...

- a13*a21*a34*a42 - a13*a22*a31*a44 - a13*a24*a32*a41...

+ a14*a21*a33*a42 + a14*a22*a31*a43 + a14*a23*a32*a41...

- a14*a21*a32*a43 - a14*a22*a33*a41 - a14*a23*a31*a42)

$\%$ NOTE: Leibniz is central for all $\operatorname{det}(\mathrm{A})$-calculations by Sarrus and by Laplace 
$\% 3 \times 3$ matrices calculated by Sarrus Rule directly from Leibniz Formula

$\%$ nxn matrices $(n=3,4, \ldots)$ by Laplace Rule directly from Leibniz, too

$\%$ Both rules of Sarrus and Laplace are visualised structures of the

$\%$ Leibniz Formula detA_Leibniz (above shown for 4x4-Matrices)

$\%$ This Leibniz Formula is ingenious as basis for Sarrus, Laplace etc

From equation (2) following equation (3) can be derived.

It is possible to expand a determinant along a row or column using this formula, which is efficient for relatively small matrices. To do this along row $i$, say, we write:

$$
\operatorname{det}(A)=\sum_{j=1}^{n} A_{i, j} C_{i, j}=\sum_{j=1}^{n} A_{i, j} \cdot(-1)^{i+j} \cdot M_{i, j}
$$

where the $C_{i, j}$ represents the $i, j$ element of the matrix cofactors, i.e. $C_{i, j}$ is $(-1)^{i+j}$ times the minor $\boldsymbol{M}_{i, j}$, which is the determinant of the matrix that results from $A$ by removing the $i$-th row and the $j$-th column, and $n$ is the length of the matrix.

The determinant of a $2 \times 2$ matrix $\mathrm{A}$ is calculated by:

$$
\begin{gathered}
M=\left[\begin{array}{ll}
a_{11} & a_{12} \\
a_{21} & a_{22}
\end{array}\right] \quad \operatorname{det}(A)=\left\|\begin{array}{ll}
a_{112} & a_{12} \\
a_{21} & a_{22}
\end{array}\right\| \\
\operatorname{det}(A)=\left(a_{11} \cdot a_{22}-a_{21} \cdot a_{12}\right)
\end{gathered}
$$

For a of $3 \times 3$ matrix, the determinant is calculated by using the Sarrus method, derivated from Leibniz's formula:

$$
\begin{aligned}
& A=\left[\begin{array}{lll}
a_{11} & a_{12} & a_{13} \\
a_{21} & a_{22} & a_{23} \\
a_{31} & a_{32} & a_{33}
\end{array}\right] \quad \operatorname{det}(A)=\left\|\begin{array}{lll}
a_{11} & a_{12} & a_{13} \\
a_{21} & a_{22} & a_{23} \\
a_{31} & a_{32} & a_{33}
\end{array}\right\|
\end{aligned}
$$

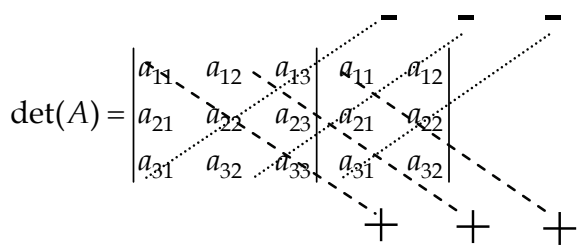

$$
\begin{aligned}
& \operatorname{det}(A)=\left(a_{11} \cdot a_{22} \cdot a_{33}+a_{12} \cdot a_{23} \cdot a_{31}+a_{13} \cdot a_{21} \cdot a_{32}-a_{31} \cdot a_{22} \cdot a_{13}-a_{32} \cdot a_{23} \cdot a_{11}-a_{33} \cdot a_{21} \cdot a_{12}\right)
\end{aligned}
$$

To find the determinant of a $3 \times 3$ matrix according the Laplace formula shown in the equation (3):

$$
\operatorname{det}(A)=\left\|\begin{array}{lll}
a_{11} & a_{12} & a_{13} \\
a_{21} & a_{22} & a_{23} \\
a_{31} & a_{32} & a_{33}
\end{array}\right\|=a_{11}\left\|\begin{array}{ll}
a_{23} \\
a_{32}
\end{array}\right\|-a_{12}\left\|\begin{array}{ll}
a_{23} & a_{23} \\
a_{31} & a_{33}
\end{array}\right\|+a_{13}\left\|\begin{array}{ll}
a_{32} & a_{22} \\
a_{31} & a_{22}
\end{array}\right\|
$$




$$
\operatorname{det}(A)=\left\{a_{11}\left(a_{22} \cdot a_{33}-a_{32} \cdot a_{23}\right)-a_{12}\left(a_{21} \cdot a_{33}-a_{31} \cdot a_{33}\right)+a_{13}\left(a_{21} \cdot a_{32}-a_{31} \cdot a_{22}\right)\right\}
$$

Because it is dealing with a $3 \times 3$ matrix, it sets up the $3 \times 3$ sign matrix. This is always a "checkerboard" matrix that begins with a " + " sign in the upper left corner and then alternates signs along rows and columns.

The Leibniz formula is the root of the Sarrus formula and the Laplace formula. The regularity of the Leibniz-, Laplace-, and Sarrus-determinant calculation was the basis for the main author developing magic matrices and cubes through visualised transformation of the cell contents (shifting, rotating and reflecting or mirroring).

\section{Computer solution}

It was only possible to solve a $4 \times 4 \times 4$ cube (76 equations with 64 unknown) by using a several computers, working in parallel, and based on the backtracking algorithm method. This was shown by the German mathematician Walter Trump in year 2004.

\section{New Idea}

The $4 \times 4 \times 4$ cube (76 equations with 64 unknown) will be solved now by using only logical thinking and geometrical methods (bending surfaces). Assume a box, with $4 \times 4$ cells on each side is opened into a 2 dimensional plane as shown in the Figure 4; the number must be match each other to the side plane. The following sequence is used to solve the magic-matrix and cube respectively.

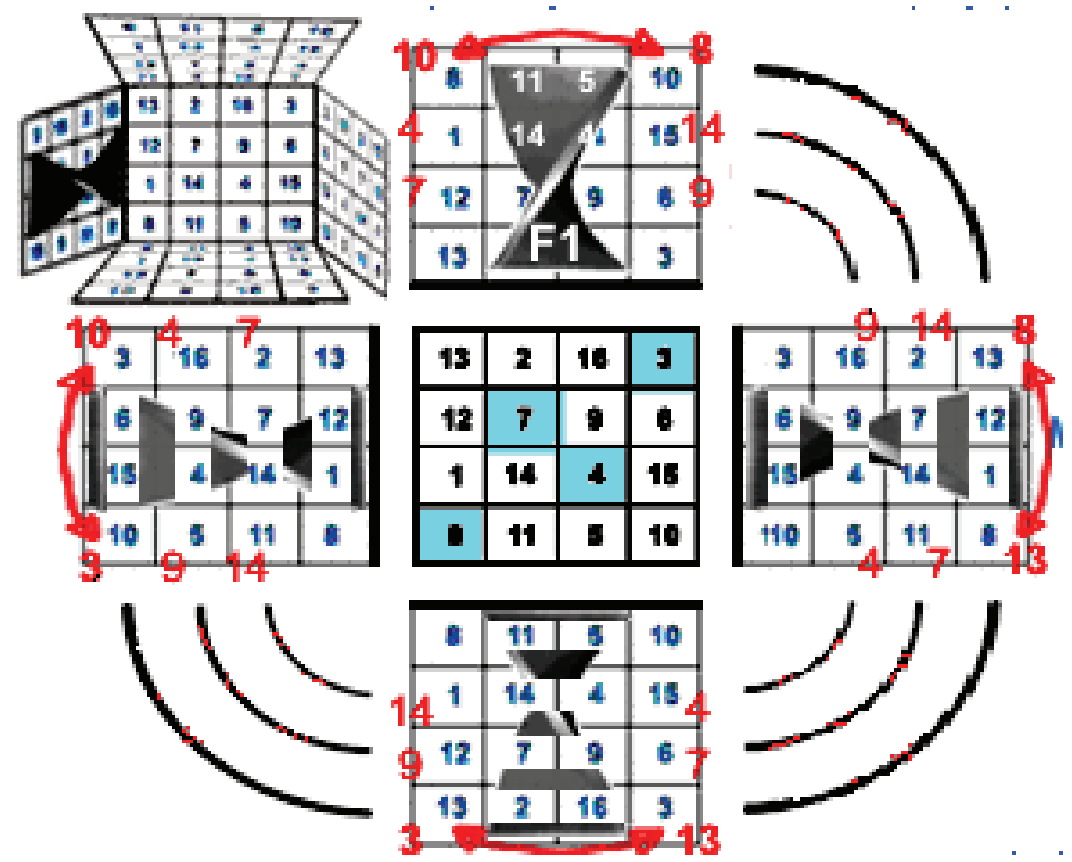

Fig. 4. Solution idea based on "box-exploding” (Stanek, 2009) 
Step 1. Start with any magic $4 \times 4$ matrix M1,

Step 2. M1 is reflected in all sides of the box.

Step 3. Use the logic method, match all the edge cells

Step 4. From M1 until M4, magic cube 2 is constructed using surface transformation, bending, or reflecting (mirroring).

The computer based magic cube solution, which is discovered in 2004 with highest degree of perfection is first in 2008 analytically solved by the main author. The solution ideas and the important steps using the Stanek Method to solve the magic cube will be shown in the following pages and a pattern solution is attached.

\section{Short information: magic cube with Stanek-Method analysis}

In the above shown graphical method the solution of magic cube is explained.

All related data of "Sudoku to the power of 3“application will be simple and always enough to solve or to construct in the 4 main layers.

\subsection{Example: magic + ultra-magic square and cube}

A magic square is bi-magic (or multi-magic) if it remains magic after each of its number has been squared and an ultra magic square has more extended properties. The following $4 \times 4$ square shows an example of ultra magic square (Fig. 5.(a)).

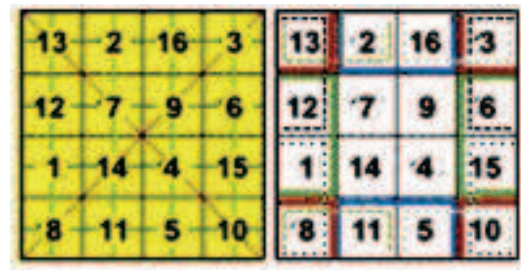

\section{Example of ultramagic Matrix sum of 4 number clusters always constant. 34}

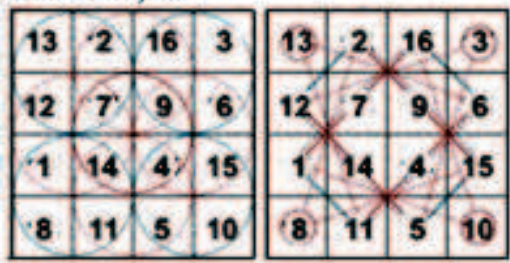

(a)

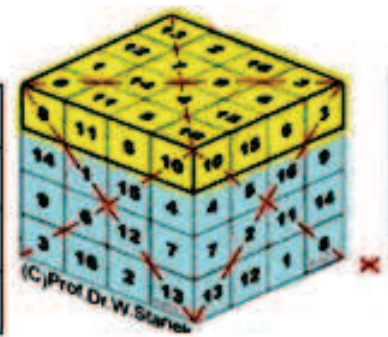

(b)

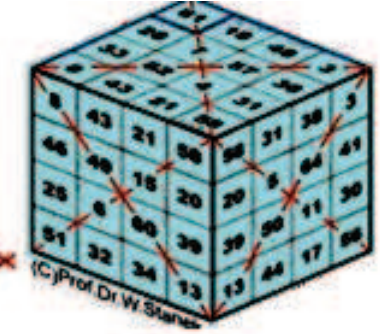

(c)

Fig. 5. (a) Perfect square or ultra-magic square with continuous number 1 to 16 all rows, columns, diagonals, and four neighbor cells always resulting magic sums $=34$

(b) Magic Cube developed from magic square, with continuous number 1 to 16 in 3D, resulting a perfect magic sum $=34$ in rows, columns, diagonals

(c) Magic Cube developed from magic square, with number 1 to 64 in 3D, resulting a perfect magic sum=130 in rows, columns, diagonals in $x-, y-$, and z-planes (Stanek, 2009) 
The sum of all numbers in the horizontal, the vertical as well as in the diagonals are equal and also the sum of all four neighbor cells, which form a square are always constant to 34 . The square pattern in Duerer's square and Loh Shu's square are easy to memorised. Starting with predetermined Loh Shu's pattern, a 9x9 squares can be constructed easily by applying a shifting, reflecting method.

A magic cube with $4 \times 4 \times 4$ dimension can be developed for instance from the known Duerer's square pattern, or extended from ultra magic square, converted by reflecting, shifting and bending.

\subsection{An ultramagic square as the base of Stanek Cube Developments}

Each of 48 given ultra-magic matrices or squares results in a new magic cube with a maximum possible degree of perfection. Through simple transformation, (shifting, rotating and reflecting) it is easy to construct other ultra magic matrices.

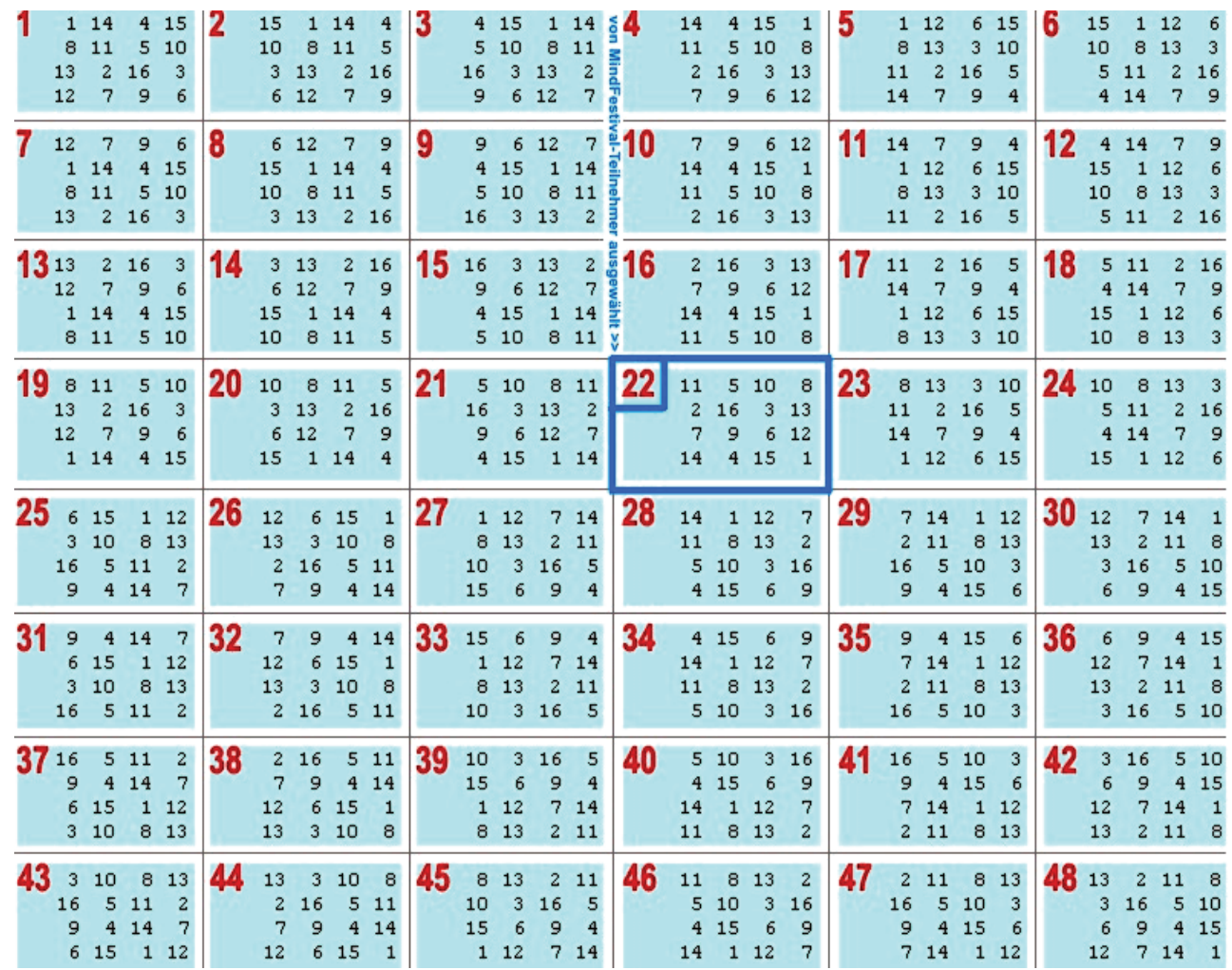

Fig. 5. 48 possible magic squares, constructed through shifting, rotating and reflecting.

\section{Solution by using mnemonic scheme for Stanek Cube No.1 + No.2}

On the Fig. 4 it is shown how to generate and to develop a magic square and magic cubes from a given ultra-magic square. An example is the square number 22 shown in the Fig. 5. 
(given by the audience at MemoMasters - MindFestival 2009 to Prof. W. Stanek for manual solution). Starting by choosing this ultra magic square number 22 as the start matrix M1, the next ultra magic square M2 is created by using the reflecting (mirroring) method. Then from matrix M2 we can construct the next matrix M3 by transposing and at the same time reflecting the content of cells and finally the matrix M4 is generated from M3 using reflecting in each four neighbor cells (number 1 to 16).

The resulting ultra-magic matrices M1 until M4 are used to develop the next layers; creating the magic cube layers with a sketching pattern $(+0,+16,+32,+48)$, as shown in the Fig.6.
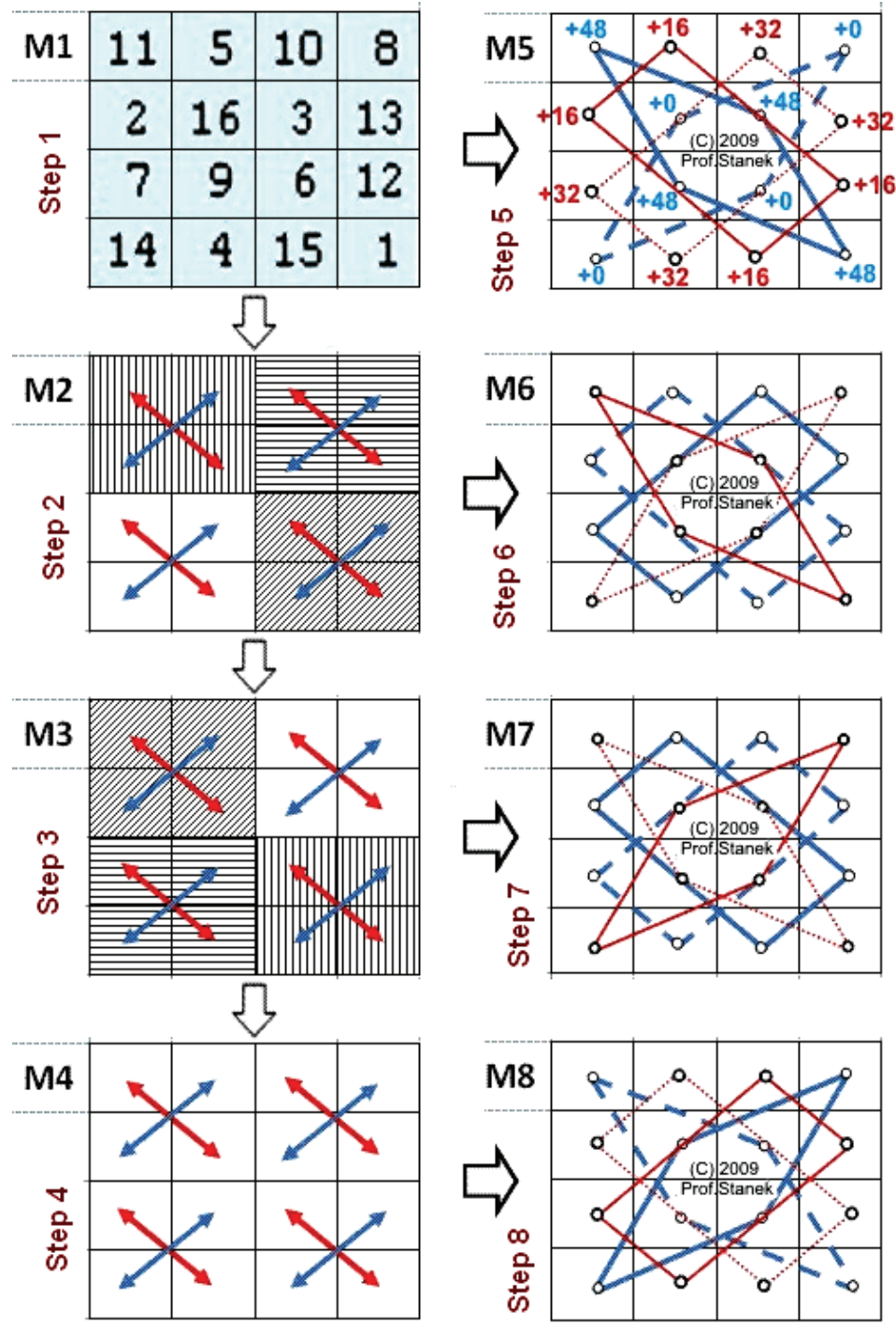

Fig. 6. Pattern Solution to solve a magic cube (Stanek, 2009) 


\subsection{First comparison: computer and analytical solution with highest degree of perfection}

A comparison between a solution which was reached by using computer backtracking method (W. Trump, 2004) and an analytical solution using logic and brain memory shows that the Stanek analytical solution delivers the highest perfect precision of magic cube (MemoMasters 2008).
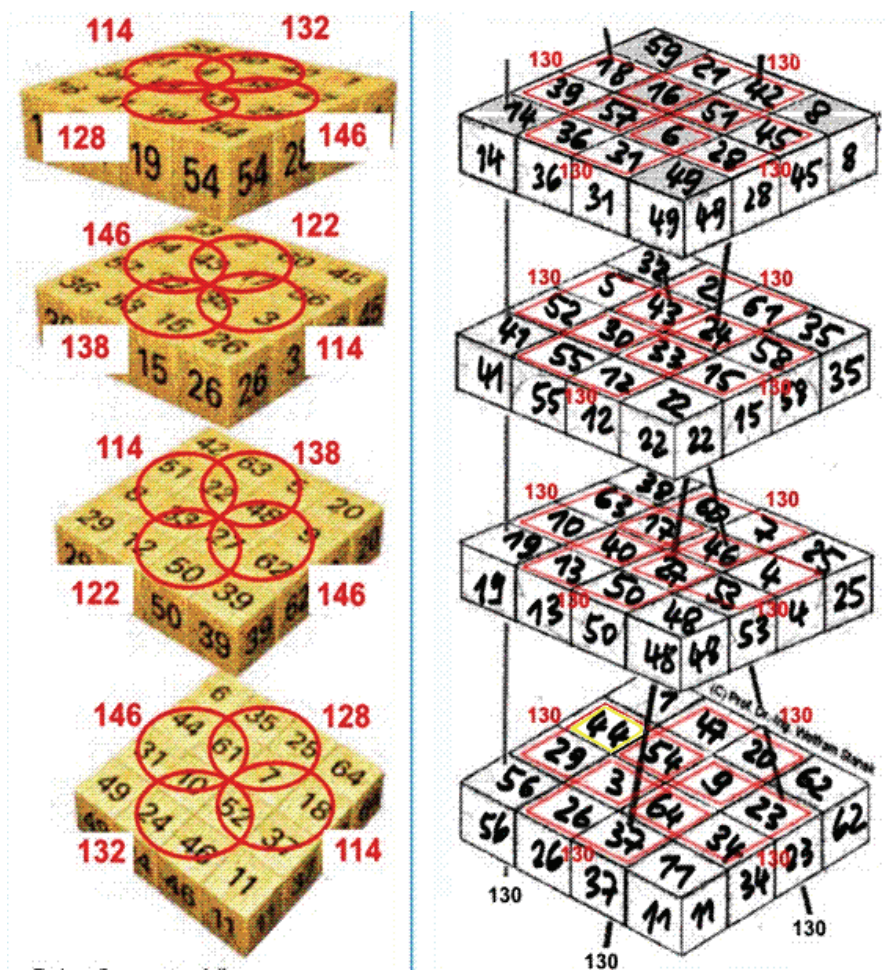

Fig. 7. (a) Magic cube, computer solution by W.Trump (Spektrum Wissenschaft, 2008) (b) Analytical solution by Prof. Wolfram Stanek.

This result for the specific analytical cube was predictable, since a start ultra-magic matrix was chosen. The result in Fig. 7.(a) was shown in "Spektrum der Wissenschaft", 2008 and the Fig. 7.(b) was represented on MindFestival in August 2009. In all the transformations of planes in the space partially some magical sum properties are lost. If many magical properties in the initial matrix are available (at ultra magic matrix of the case), the result of the magic cube (1-64) has a relatively highest degree of perfection.

\subsection{Second comparison: minimising the deviation of space diagonals with computer and analytical methods}

By choosing the Duerer's matrix, which is not perfect, a magic matrix is created as the start matrix in developing other magic cubes as shown in the Fig. 8.

The Fig.9 shows the difference between W.Trump's magic cube which is solved by computer and the main author's analytical solution by starting with the magic matrix in the 
previous Fig. 8. The gray areas in the figure represent the difference between both solutions. In the right column is shown the magic cube, which is constructed through twisted matrix transformation. The highlighted cells (rows, column and diagonals) produce the constant sum of the magic cube.

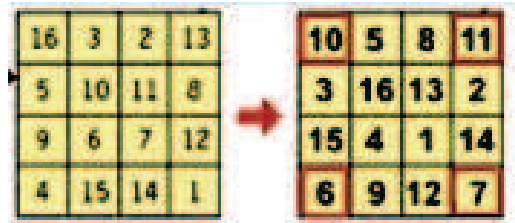

Fig. 8. Constructing a magic cube, starting with Duerer's matrix (Stanek, 2009)

Computer Solution by W.Trump:

\begin{tabular}{|c|c|c|c|}
\hline 58 & 21 & 40 & 11 \\
\hline 19 & 16 & 61 & 34 \\
\hline 47 & 52 & 1 & 30 \\
\hline 6 & 41 & 28 & 55 \\
\hline
\end{tabular}

$x-y$ : Mag. Matrix M2 $(v, 12)$

\begin{tabular}{|c|c|c|c|}
\hline 17 & 15 & 62 & 36 \\
\hline 8 & 42 & 27 & 53 \\
\hline 60 & 22 & 39 & 9 \\
\hline 45 & 51 & 2 & 32 \\
\hline
\end{tabular}

$x \cdot y$. Mag. Matrix M3 $(v, 12)$

\begin{tabular}{|c|c|c|c|}
\hline 48 & 50 & 3 & 29 \\
\hline 57 & 23 & 38 & 12 \\
\hline 5 & 43 & 26 & 56 \\
\hline 20 & 14 & 63 & 33 \\
\hline
\end{tabular}

$x \cdot y:$ Mag. Matrix Mu (v, 12)

\begin{tabular}{|c|c|c|c|}
\hline 7 & 44 & 25 & 54 \\
\hline 46 & 49 & 4 & 31 \\
\hline 18 & 13 & 64 & 35 \\
\hline 59 & 24 & 37 & 10 \\
\hline
\end{tabular}

Analytical Solutions with Stanek-Method

\begin{tabular}{|c|c|c|c|}
\hline 58 & 21 & 40 & 11 \\
\hline 19 & 16 & 61 & 34 \\
\hline 47 & 52 & 1 & 30 \\
\hline 6 & 41 & 28 & 55 \\
\hline
\end{tabular}

x-y: Mag.Matrix M2 (v. 12)

\begin{tabular}{|c|c|c|c|}
\hline 32 & 3 & 50 & 45 \\
\hline 5 & 42 & 27 & 56 \\
\hline 57 & 22 & 39 & 12 \\
\hline 36 & 63 & 14 & 17 \\
\hline
\end{tabular}

x.y. Mag.Matrix M3 (v, 12)

\begin{tabular}{|c|c|c|c|}
\hline 33 & 62 & 15 & 20 \\
\hline 60 & 23 & 38 & 9 \\
\hline 8 & 43 & 26 & 53 \\
\hline 29 & 2 & 51 & 48 \\
\hline
\end{tabular}

$x-y:$ Mag.Matrix Ma (v, 12)

\begin{tabular}{|c|c|c|c|}
\hline 7 & 44 & 25 & 54 \\
\hline 46 & 49 & 4 & 31 \\
\hline 18 & 13 & 64 & 35 \\
\hline 59 & 24 & 37 & 10 \\
\hline
\end{tabular}

\begin{tabular}{|c|c|c|c|}
\hline 26 & 53 & 8 & 43 \\
\hline 51 & 48 & 29 & 2 \\
\hline 15 & 20 & 33 & 62 \\
\hline 38 & 9 & 60 & 23 \\
\hline
\end{tabular}

$x-y$, Mag. Matrix M2 $(v, 12)$

\begin{tabular}{|c|c|c|c|}
\hline 64 & 35 & 18 & 13 \\
\hline 37 & 10 & 59 & 24 \\
\hline 25 & 54 & 7 & 44 \\
\hline 4 & 31 & 46 & 49 \\
\hline
\end{tabular}

$x-y$ : Mag. Matrix M3 $(v, 12)$

\begin{tabular}{|c|c|c|c|}
\hline 1 & 30 & 47 & 52 \\
\hline 28 & 55 & 6 & 41 \\
\hline 40 & 11 & 58 & 21 \\
\hline 61 & 34 & 19 & 16 \\
\hline
\end{tabular}

$x-y:$ Mag-Matrix M4 (v. 12)

\begin{tabular}{|c|c|c|c|}
\hline 39 & 12 & 57 & 22 \\
\hline 14 & 17 & 36 & 63 \\
\hline 50 & 45 & 32 & 3 \\
\hline 27 & 56 & 5 & 42 \\
\hline
\end{tabular}

Fig. 9. Complete solution to build magic matrices and magic cube with highest symmetry (Stanek, 2009)

The comparison also shows that the Stanek analytical method works magically not only in start-matrix of ultra-magic, but also in normal magical start squares, which have at least partly-some pandiagonal magic properties. In Duerer's matrix the major side-diagonals (e.g. $2+10+9+1=22$, etc) are not magical, but parts of the side-diagonal always have a magic constant (e.g. $3+5+14+12=34$, etc). 


\section{New magic matrix applications for interdisciplinary system design}

Now, the question is: "Is this a mathematical phenomenon, or what we see and what we need?" A main question for our brain and our life is what is important and not important for us temporarily? According to "Spektrum der Wissenschaft, 2008-2", there is no existing absolutely perfect cube $4 \times 4 \times 4$ because the main spatial diagonal always deviates.

Is it correct or is it a starting question? A near perfect $4 \times 4 \times 4$ cube is shown in the Fig.10 (Nintendo MemoMasters 2009 -MindFestival)

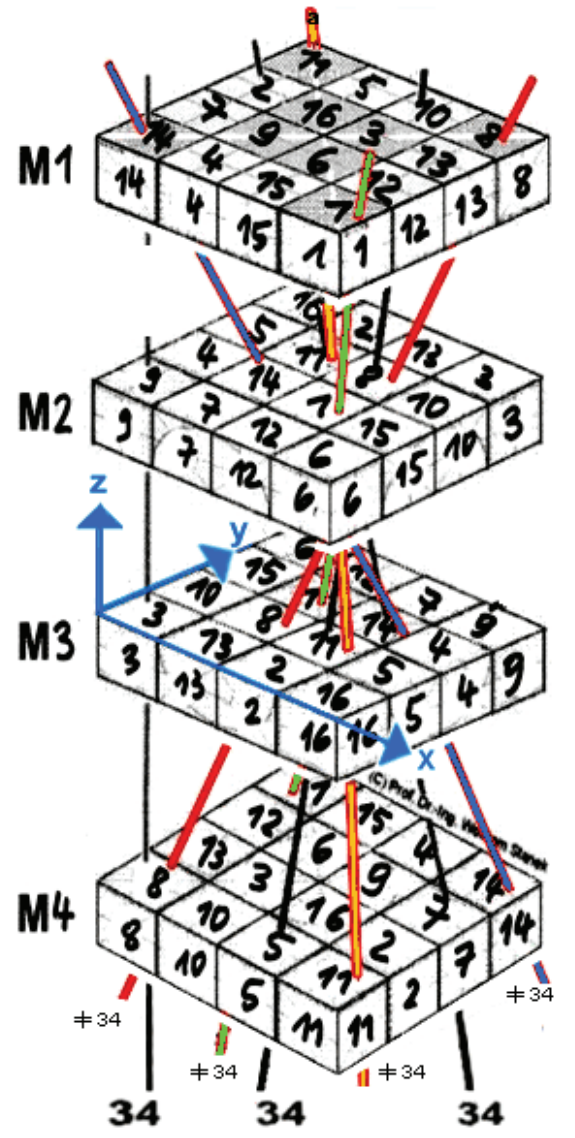

(a)

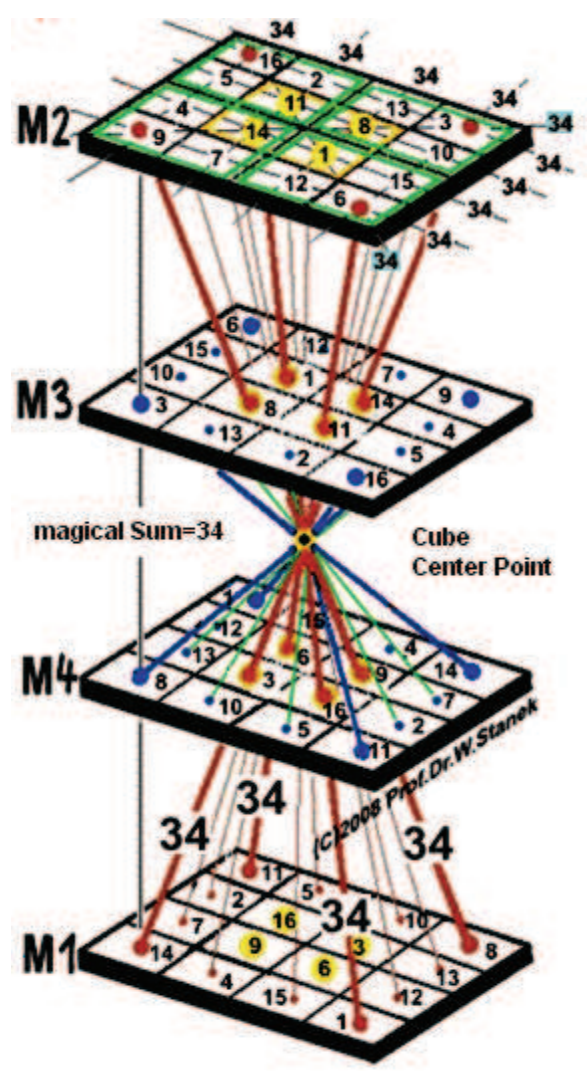

(b)

Fig. 10. (a). Near perfect magic cube (b) Perfect magic cube (Stanek, 2009)

The only once calculated building block - depending on the "Position" -surprisingly contains quite different symmetry results.

- In the Fig. 10.(a): in all mutually perpendicular surfaces of magic numbers (symmetry) there is 'perfect Cartesian', but not in the spatial diagonals.

- In the Fig. 10.(b): just by shifting the layer M1 "perfect central symmetry" is achieved. All major and minor diagonals have a constant sum of 34 (suggestion by Einstein "... all is relative") 


\subsection{Strategy-checks "Sudoku to the Power of X" + scientific and technological point of view.}

(A) "Sudoku to the power of 3"with ultra-magic Start-squares \& Stanek-Method.

1. Constructing 11 new magic squares from 1 ultra magic square; select any ultra magic square from Fig. 5 on page 7 (manually, required time: less than 90 seconds)

2. Constructing near perfect magic cube no. 1 (number 1 to 16) from point A.1.

3. Constructing any possible perfect magic cube no.2 (number 1 to 64 ) from point A.2 Notes:

Until 2004 this mathematical problem was thought to be unsolvable, then in 2004 it was solved by Trump using parallel computing of computers and backtracking method; in 2008 this problem was solved analytically and finally in 2009 it is solved by using the Stanek-Pattern Strategy during MemoMasters 2009.

For Strategy B and Strategy C improvement, try your own "creative intelligence " in logic and mathematic areas.

Try to select a solution strategy from the following improvement that you can find quickly.

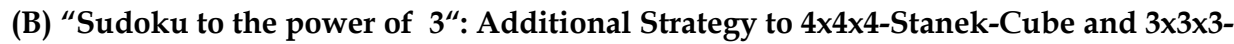
Rubik-cube

1. Try to find an extension strategy for a Stanek magic cube with a given sum value greater than 130; example the magic sum equals 274 and the maximal number in the cube equals 100 .

2. Construct with this extension (just addition) the new cube directly from point A.3

3. Try to apply the Stanek-strategy to generate an arbitrary sum-number (see page 5) for the color rubik cube (divisible by 3 ).

(C) "Sudoku to the power of 2 " = Combination of Sudoku and interesting special magic squares

1. Constructing a magic $4 \times 4$-Sudoku $X$ with four $2 \times 2$-fields (with each number $1-4$ )

2. Apply the $3 \times 3$-Loh-Shu-square, included design rule, so that each odd-square $n \times n$ ( $\mathrm{n}=3,5,7,9, \ldots$ etc) can be constructed immediately.

Note: Position of Serial numbers 1-9 including the solution scheme. Start-number 1 is always above in the middle, No. 2 in the right column below.

3. Construct a 9x9 magic square $\mathrm{Y}$ with this method from C.2: All rows, columns and main diagonals have a constant sum

$$
\mathrm{S}=\mathrm{n} \times(\mathrm{n} \times \mathrm{n}+1) / 2 \text { for number from } 1 \text { to } \mathrm{n}^{2} .
$$

Example, $n=9$, then $S=396$, etc.

For magic cube magic sum

$$
\mathrm{S}=\mathrm{n} \times(\mathrm{n} \times \mathrm{n} \times \mathrm{n}+1) / 2 \text {, for number from } 1 \text { to } \mathrm{n}^{3}
$$

example $4 \times 4 \times 4$ - cube, $n=4$ then $S=130$, etc

4. Try to prepare a $4 \times 4$ magic square (magic or ultra-magic square) so that for any number, the sum of all numbers in all rows, columns and main diagonals are equal.

5.a). Construct a 9x9-magic Sudoku A from a 3×3-Loh-Shu-square.

5.b). Construct a 9x9-magic Sudoku B from Sudoku A in 5.a). 
5.c). Construct bi-magic square C (Number 1 to 81) from Sudoku A and Sudoku B in 5.a) and 5.b).

5.d). Construct a new magic square $D$, where all the 81 number of square $C$ in 5.c) are squared.

\section{NOTE:}

a. Magic "Sudoku to the power of 3 " cube number 1 and 2 is relatively easy to solve by using Stanek's predetermined pattern Strategy-Method; otherwise, without this strategy the cube can be only solved by using a computer program.

b. With the Stanek-Method, a linear equation system (76 equations with 64 unknown) is solved primarily with logic and geometry.

c. The mathematical genius, physicist and astronomer, Galileo Galilei (1564-1642), said, "Who understands geometry can understand the world"

d. Problem with difficult creativity are not possible to be solved without strategy, even with best memory.

e. Test your own understanding strategy using the "Sudoku to the power of $X^{\text {" }}$ - Checks (B) \& (C), and your temporary capability for Logic-Mathematic Strategy in interdisciplinary area "Creative Intelligence".

\section{Application range of "Magic Cube" in Science and Technology.}

This is a short overview of mostly unused fantastic magic application with high precision in matrix-areas in 2D and 3D in Science and Technology Point of View.

Aspects of R\&D in engineering with focus on mechatronics and integrated interdisciplinary differential equations are extended matrix operations with magic matrix techniques too.

Unknown applications of magic matrix could be:

1. Optimised magic distribution of energy, power, element properties, information fluxes, etc. constant in all directions in 2D or 3D.

2. Automatic Minimising of energy losses in all directions in 2D or 3D.

3. Direct compensation of punctual disturbances with unchanged sum of energy

4. Optimised logistic automation and transport with different motors.

5. Magic matrices as explanation of chemical structures or unsolved problems in physics.

6. A quick and new way in solving undetermined systems of equations without using conventional method (iterative solvers and backtracking method).

\subsection{Tests of Stanek Algorithm for magic $4 \times 4 \times 4$-cubes}

To understand the analytical algorithm of magic $4 \times 4 \times 4$ cube, the following sequence is used to develop a magic cube;

Step 1. Create matrix M1, from the $4 \times 4$ magic matrix i.e. numbers 22 given in the Fig. 5.

$$
M 1=\left[\begin{array}{cccc}
11 & 5 & 10 & 8 \\
2 & 16 & 3 & 13 \\
7 & 9 & 6 & 12 \\
14 & 4 & 15 & 1
\end{array}\right] \quad M 5=\left[\begin{array}{cccc}
59 & 21 & 42 & 8 \\
18 & 16 & 51 & 45 \\
39 & 57 & 6 & 28 \\
14 & 36 & 31 & 49
\end{array}\right]
$$

Step 2. Construct the matrix M2 from M1 using reflection of the cells content diagonally (the four neighbor cells of magic matrix) according to the pattern solution shown in the Fig. 6. 


$$
M 2=\left[\begin{array}{cccc}
16 & 2 & 13 & 3 \\
5 & 11 & 8 & 10 \\
4 & 14 & 1 & 15 \\
9 & 7 & 12 & 6
\end{array}\right] \quad M 6=\left[\begin{array}{cccc}
32 & 2 & 61 & 35 \\
5 & 43 & 24 & 58 \\
52 & 30 & 33 & 15 \\
41 & 55 & 12 & 22
\end{array}\right]
$$

Step 3. Developing the matrix M3 from M2 by reflection of the internal four neighbor cells diagonally.

$$
M 3=\left[\begin{array}{cccc}
6 & 12 & 7 & 9 \\
15 & 1 & 14 & 4 \\
10 & 8 & 11 & 5 \\
3 & 13 & 2 & 16
\end{array}\right] \quad M 7=\left[\begin{array}{cccc}
38 & 60 & 7 & 25 \\
63 & 17 & 46 & 4 \\
10 & 40 & 27 & 53 \\
19 & 13 & 50 & 48
\end{array}\right]
$$

Step 4. Developing the matrix M4 from M3 by using reflection of the cells content diagonally (the four neighbor cells of magic matrix), according to the pattern solution shown in the Fig. 6 and similar to the step 2.

$$
M 4=\left[\begin{array}{cccc}
1 & 15 & 4 & 14 \\
12 & 6 & 9 & 7 \\
13 & 3 & 16 & 2 \\
8 & 10 & 5 & 11
\end{array}\right] \quad M 8=\left[\begin{array}{cccc}
1 & 47 & 20 & 62 \\
44 & 54 & 9 & 23 \\
29 & 3 & 64 & 34 \\
56 & 26 & 37 & 11
\end{array}\right]
$$

\section{NOTES:}

- $\quad$ The matrices M1 until M4 are magic-matrices with the cells content of number 1 to 16 .

- $\quad$ To construct 3D magic cube, layer 1 (M5), layer 2(M6), layer 3 (M7) and layer 4 (M8) are developed from M1, M2, M3 and M4 respectively by using mnemotechnique scheme solution as shown in the Fig. 6.

The Fig. 11 shows a sample of MATLAB® program listing as a test algorithm of a magic $4 \times 4 \times 4$ cube which is tested by Martin Eka Putra and Jemmy Dianto (SGU students) during internship in Germany, and F. Halfmann (Assistant in Lab Automation \& Robotics FH Koblenz) and then the magic sum is represented graphically in the next Fig. 12. It can be clearly seen that the continuous numbers from 1 to 64 fit in the cube are without any repetition. Fig. 13 shows the sum of the columns, rows, and main diagonals in 3D equal to 130 and the spatial diagonals' sum also has symmetry.

\section{Application of the magic matrix transformation in Science and Technology}

Now, we can apply the concept of the magic matrix in 2D as well as in 3D into the real science and technology field. Let a production system consisting of four rows of transport mechanism (e.g. using conveyors) be assumed as a matrix. Each of conveyors are divided into four parts and driven by four electric motors, as illustrated in the Fig. 14 (a). How is the energy or power in all conveyor motors with different power distributed so that all four clusters can in parallel produce products with the same power respectively energy? 


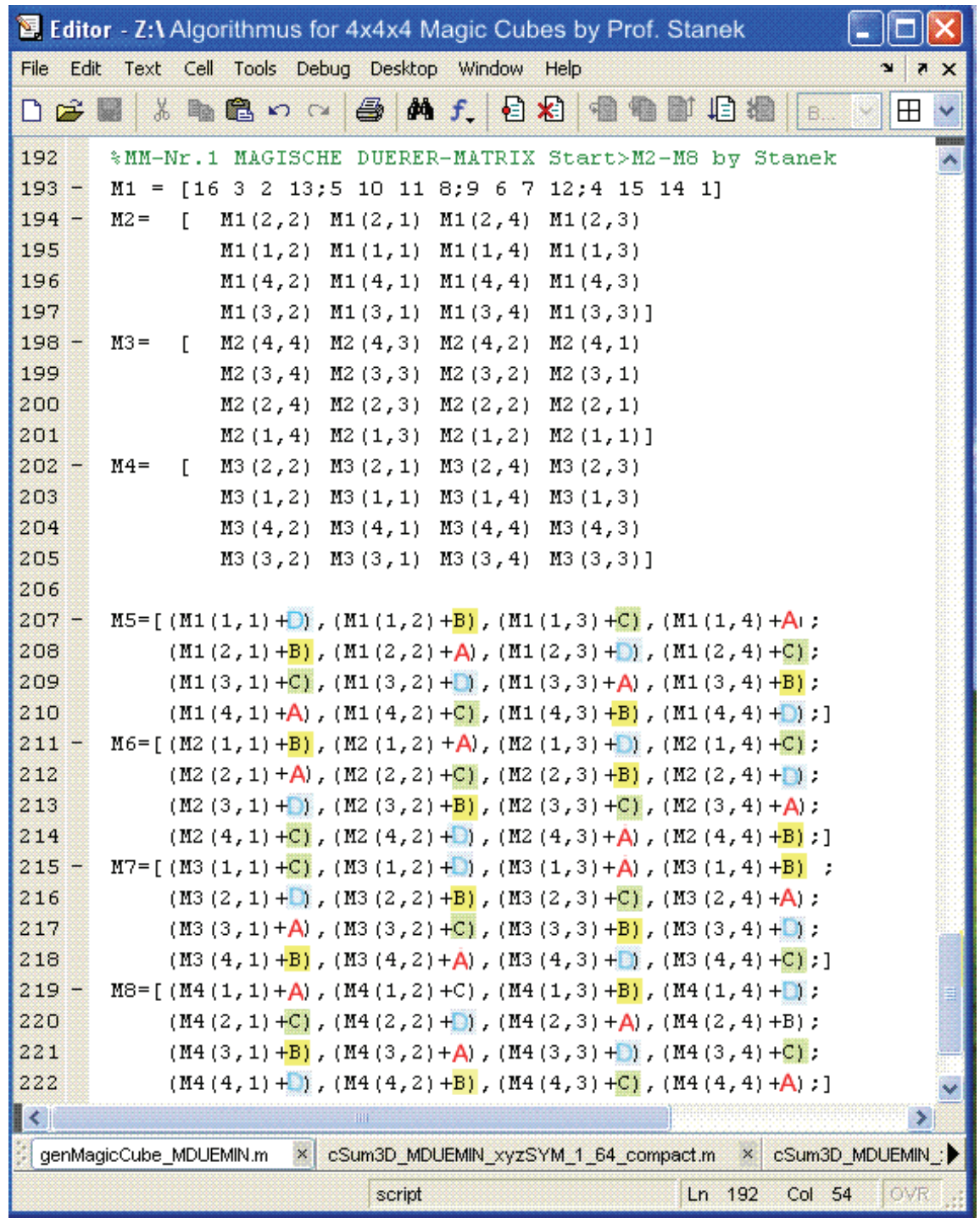

Fig. 11. MATLAB ${ }^{\circ}$ program listing for test algorithm, with $A, B, C$, D equal to $+0,+16,+32,+48$. (Stanek, 2010) 
Magic Mathematics Based on New Matrix Transformations (2D and 3D)

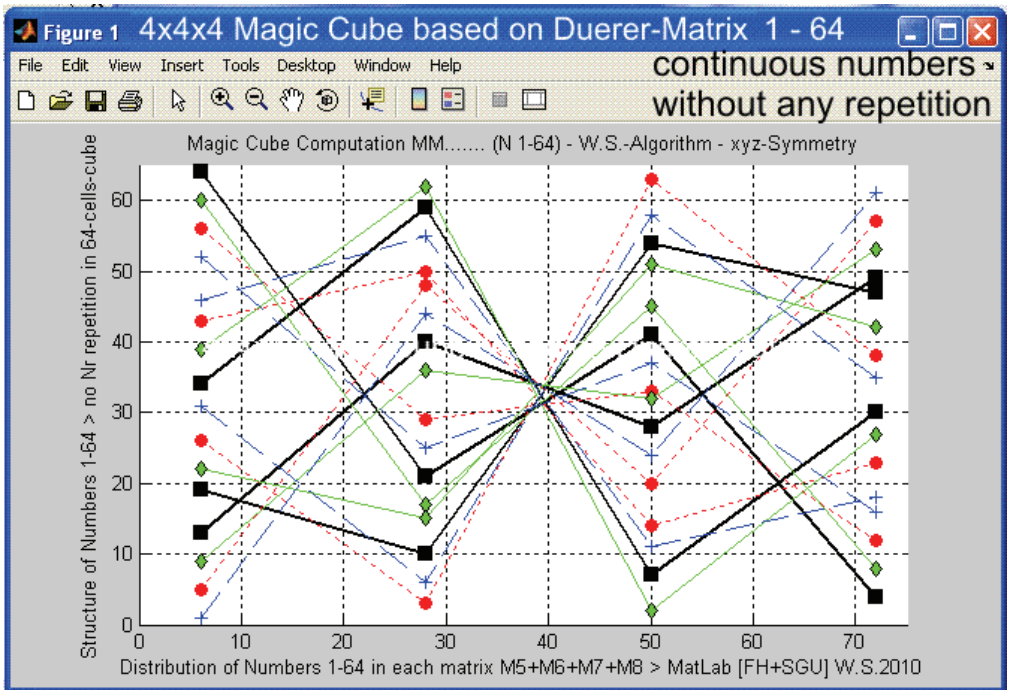

Fig. 12. Distribution of continuous number 1 to 64 in the magic cube (Stanek, 2010)

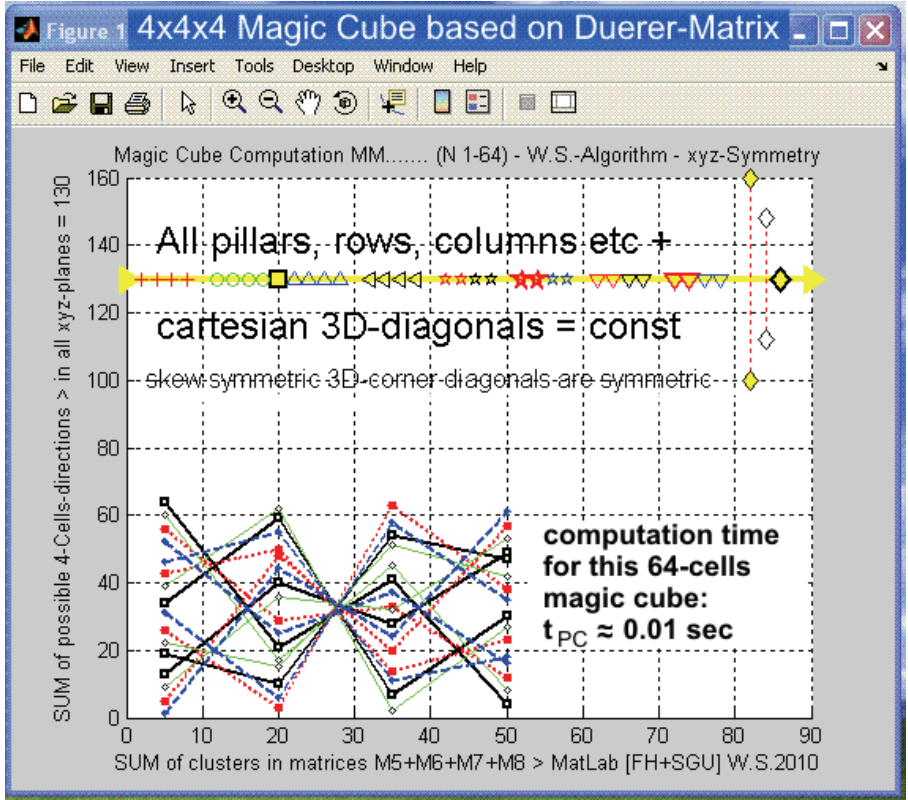

Fig. 13. Magic sum of rows, column, and diagonals (Stanek, 2010) 


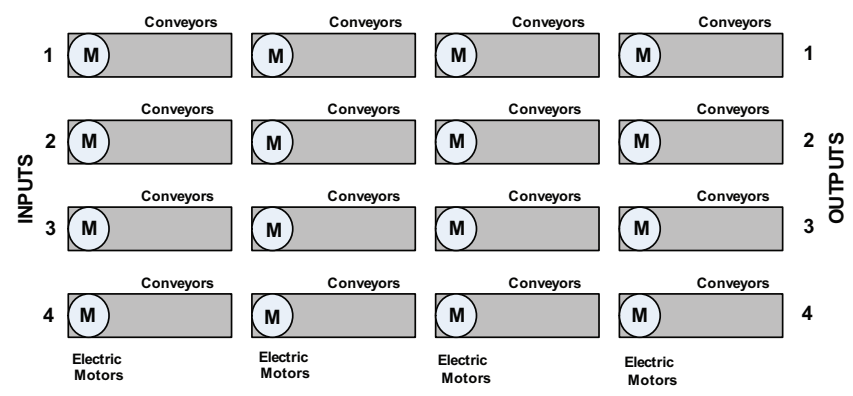

(a) Technology

\begin{tabular}{|c|c|c|c|}
\hline 16 & 3 & 2 & 13 \\
\hline 5 & 10 & 11 & 8 \\
\hline 9 & 6 & 7 & 12 \\
\hline 4 & 15 & 14 & 1 \\
\hline
\end{tabular}

(b) Continuous power

\begin{tabular}{|c|c|c|c|}
\hline 104 & 6 & 81 & 9 \\
\hline 8 & 82 & 7 & 103 \\
\hline 5 & 101 & 10 & 84 \\
\hline 83 & 11 & 102 & 4 \\
\hline
\end{tabular}

(c) Clustered power

Fig. 14. (a) Example of magic square application in the production line, with the maximum power distribution per row and column less than 200kW (Stanek, 2010)

(b) Start Matrix, i.e. Duerer matrix, (c) Constructed magic matrix, shows the distributed power in each row using i.e. 4 main power drives and smaller sub-drives, column and diagonal are constant sum of $200 \mathrm{~kW}$.

To produce a constant amount of production in any row, how is the energy and motor power respectively distributed without exceeding maximum power of i.e. $200 \mathrm{~kW}$, total maximum power consumption per conveyor-line is derived from Duerer start-matrix in Fig. 14 (b) and developed into power distribution as shown in the Fig. 14 (c).

An example of a $4 \times 4$ magic square with constant sum zero, known as zero-sum matrix, is shows in the Fig. 16.

The represented matrix is derived from a magic matrix by addition and subtracting of cell contents with a constant, so the resulting matrix has a magic constant sum zero. This can be applied to the design of coils or transformer windings as shown in the next Fig. 15.Another example is if we apply the magic square 2D to construct real 3D applications in magnetic field as shown in the Fig 15 and Fig. 16. It shows the symmetry with focus on separate $x_{-}^{-}, y^{-}$, and z-regions.

Real applications with totally different features in all cells of 3D can be optimised with constant sums in arbitrary directions using the real magic-matrix algorithm for $4 \times 4 \times 4$ cubes shown in Fig. 6 to Fig. 10.

From the Fig. 15 (a) can be seen that the spatial field is zero due to the symmetry of the magic matrix in developing of the windings currents. The sum of all four-cell-clusters is always zero. This phenomenon might be theoretically interpreted as a galaxy black hole, too. The magic square and cube could be applied also in other science and technology fields, such as energy management, flow management, logistics, and thermodynamics etc. See the spectrum of possible magic -matrix applications in section 7.1 too. 
Magic Mathematics Based on New Matrix Transformations (2D and 3D)

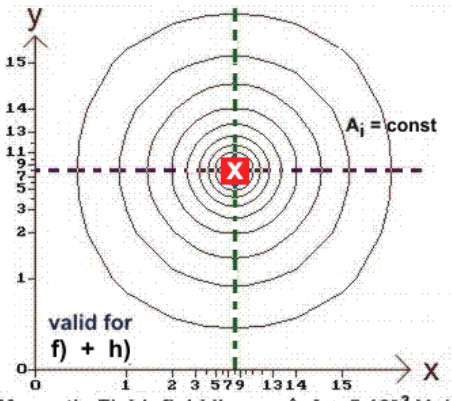

a) Magnetic Field: field lines $\triangle A=5 \cdot 10^{-3} \mathrm{Vs} / \mathrm{m}$

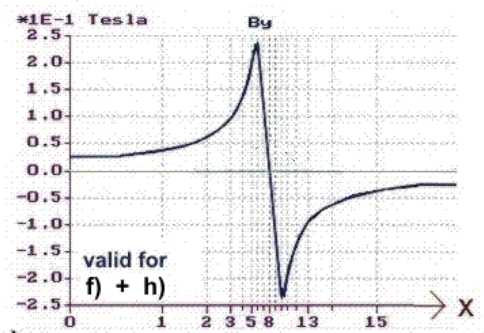

C) Magnetic Flux density B along meshes $y=8$

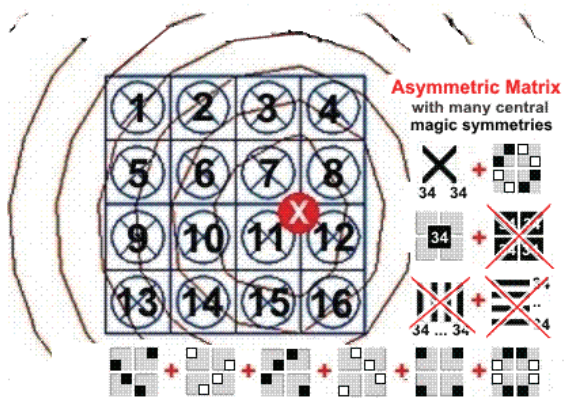

e) Magnetic Field: SEMI-magic conductor design current density in conductors $\mathrm{J}_{1.16}=1 \ldots 16 \mathrm{~A} / \mathrm{mm}^{2}$

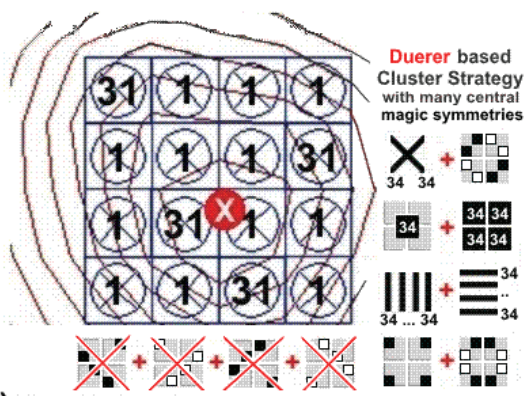

g) Magnetic Field: DUERER-magic conductor design 4 main sources +12 sub sources $\mathrm{J}=31 \& 1 \mathrm{~A} / \mathrm{mm}^{2}$

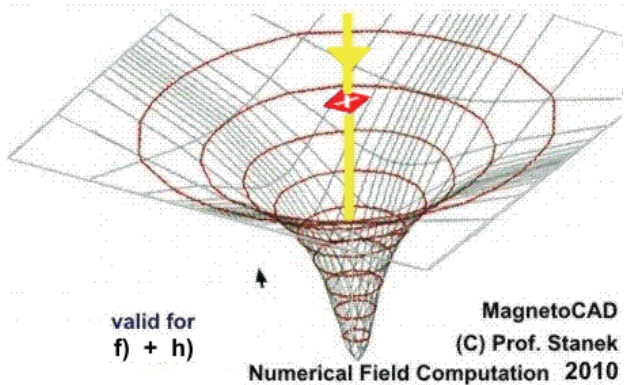

b) 3D-Magnetic Vector Potential Surface Field Fig. a)

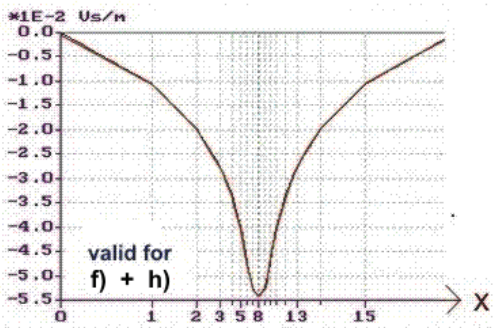

d) Magnetic Vector Potential A along points $y=8$

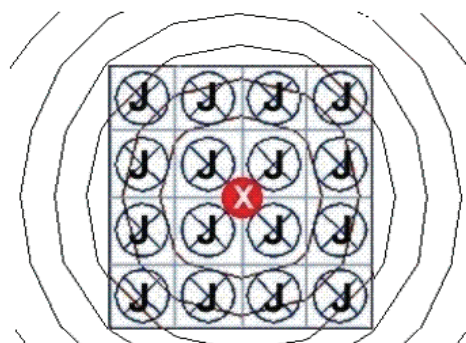

Same Results for $J_{1 . .16}=$ const \& J J $1.16 \neq$ const $/ h$ )

f) Magnetic Field: automatically "ULTRA-magic" current density in 16 conductors $\mathrm{J}_{1.16}=8.5 \mathrm{~A} / \mathrm{mm}^{2}$

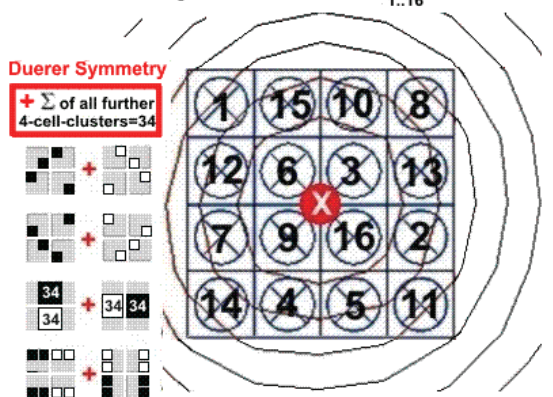

h) Magnetic Field: ULTRA-magic conductor design current density in conductors $\mathrm{J}_{1.16}=1 \ldots 16 \mathrm{~A} / \mathrm{mm}^{2}$

Fig. 15. Current windings design with semi-magic \& ultra-magic matrices 


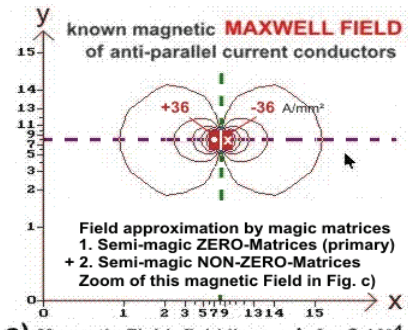

a) Magnetic Field: field lines $\triangle A=6 \cdot 10^{-4} \mathrm{Vs} / \mathrm{m}$
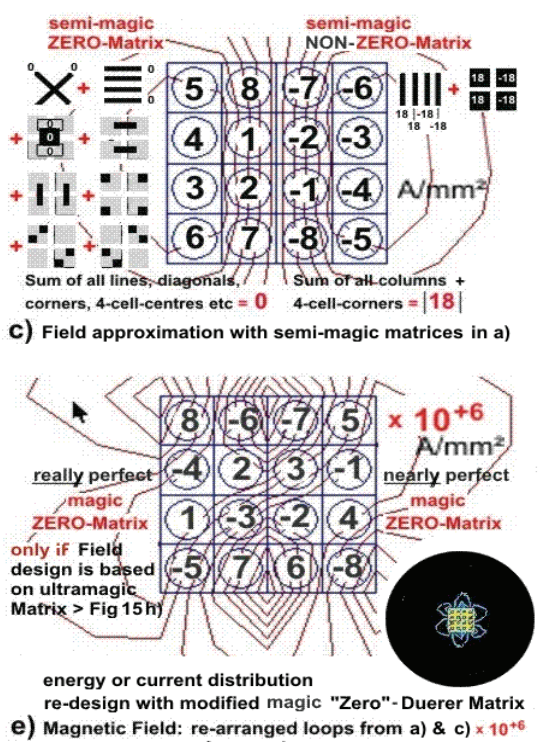

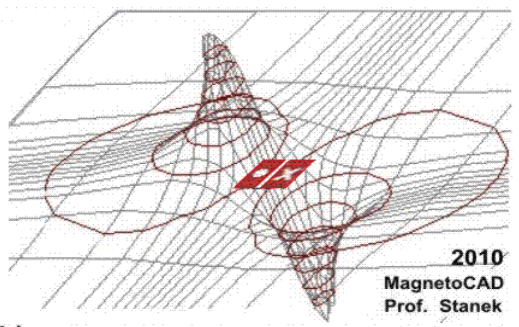

b) 3D-Magnetic Vector Potential Surface of Field Fig. a)

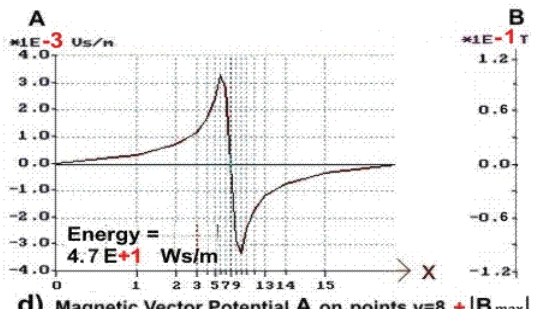

d) Magnetic Vector Potential $A$ on points $y=8+\mid B$ max $\mid$

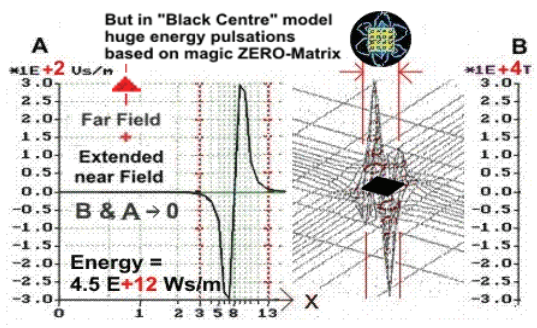

f) Magnetic Vector Potential $A+3 D$-Surface $+\mid B$ max $\mid$

Fig. 16. Current windings and field design with magic ZERO matrices

\section{Acknowledgements}

Thanks of main author to his appreciated colleague and co-author Dipl. Ing. Maralo Sinaga from SGU-Asia for adaptation and compression of central magic matrix developments based on W.Stanek's research and publication (refer list of publications).

Thanks of main author also to the SGU internship student Martin Eka Putra, Jemmi Dianto and assistant Florian Halfmann from University of Applied Sciences, Koblenz, testing the new 3D algorithm for arbitrary 64 cells magic cube with MATLAB®.

\section{References}

www.wolfram-stanek.de/stanek_sudoku_hoch_3_magisch_mindfestival2009.pdf

www.memomasters.de , "Mensch schlägt Computer"; MemoMasters 2009, 2008, Germany. Spektrum der Wissenschaft, 2008-2,

Stanek Wolfram Prof, www.wolfram-stanek.de, 2010

http://en.wikipedia.org/wiki/Magic_square

http://www.multimagie.com/bi-magic squares 


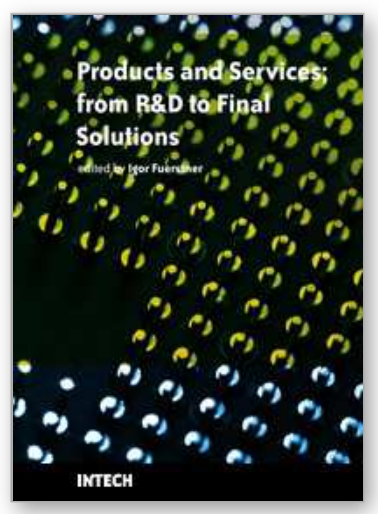

\author{
Products and Services; from R\&D to Final Solutions \\ Edited by Igor Fuerstner
}

ISBN 978-953-307-211-1

Hard cover, 422 pages

Publisher Sciyo

Published online 02, November, 2010

Published in print edition November, 2010

Todayâ $\epsilon^{\mathrm{TM}} \mathrm{s}$ global economy offers more opportunities, but is also more complex and competitive than ever before. This fact leads to a wide range of research activity in different fields of interest, especially in the socalled high-tech sectors. This book is a result of widespread research and development activity from many researchers worldwide, covering the aspects of development activities in general, as well as various aspects of the practical application of knowledge.

\title{
How to reference
}

In order to correctly reference this scholarly work, feel free to copy and paste the following:

Wolfram Stanek and Maralo Sinaga (2010). Magic Mathematics Based on New Matrix Transformations (2D and 3D) for Interdisciplinary Physics, Mathematics, Engineering and Energy Management, Products and Services; from R\&D to Final Solutions, Igor Fuerstner (Ed.), ISBN: 978-953-307-211-1, InTech, Available from: http://www.intechopen.com/books/products-and-services--from-r-d-to-final-solutions/magic-mathematicsbased-on-new-matrix-transformations-2d-and-3d-for-interdisciplinary-physics-mathem

\section{INTECH}

open science | open minds

\section{InTech Europe}

University Campus STeP Ri

Slavka Krautzeka 83/A

51000 Rijeka, Croatia

Phone: +385 (51) 770447

Fax: +385 (51) 686166

www.intechopen.com

\section{InTech China}

Unit 405, Office Block, Hotel Equatorial Shanghai

No.65, Yan An Road (West), Shanghai, 200040, China

中国上海市延安西路65号上海国际贵都大饭店办公楼 405 单元

Phone: +86-21-62489820

Fax: +86-21-62489821 
(C) 2010 The Author(s). Licensee IntechOpen. This chapter is distributed under the terms of the Creative Commons Attribution-NonCommercialShareAlike-3.0 License, which permits use, distribution and reproduction for non-commercial purposes, provided the original is properly cited and derivative works building on this content are distributed under the same license. 